\title{
Rumination and executive function in depression: an experimental study
}

\section{E Watkins, R G Brown}

Background: Major depression is associated with cognitive deficits, particularly those requiring central executive functioning. Depressed patients also tend to focus on and think about their symptoms and problems ("ruminate") more than non-depressed controls. Although an association has been found between rumination and impaired performance on a central executive processing task, the causal relation between impaired executive functioning and rumination has not been determined. This study sought to directly manipulate rumination and assess the impact on executive functioning in depression as measured by random number generation.

Methods: Depressed patients $(n=14)$ and non-depressed controls $(n=14)$ were compared on a random number generation task, performed after both a rumination induction and after a distraction induction, with order of inductions counter balanced within each group.

Results: Compared with the distraction induction, the rumination induction produced a significant increase in both ruminations and the tendency towards stereotyped counting responses (thought to reflect a failure of inhibitory executive control) in depressed patients but not in controls. However, after distraction, no difference in random number generation or rumination was found between the two groups.

Conclusions: The aspects of executive function involved in random number generation are not fundamentally impaired in depressed patients. In depressed patients, the rumination induction seems to trigger the continued generation of ruminative stimulus independent thoughts, which interferes with concurrent executive processing.

C onsistent with evidence from imaging studies showing metabolic changes in the prefrontal cortex, patients with depression often show executive dysfunction as part of a broader pattern of cognitive impairment. ${ }^{1}$ By definition, executive tasks are effortful and depend on access to limited capacity cognitive processes. Although depression may lead to a primary deficit in these processes, it is also possible that impairment on executive tasks may result from, or be exacerbated by, interference from other ongoing cognitive activity. Patients with depression typically show a range of cognitive distortions and alterations in thinking style. ${ }^{2}$ Of particular relevance is the tendency of some patients to ruminate, defined as a repetitive focusing on oneself and the nature and implications of negative feelings. ${ }^{3-5}$ Davis and NolenHoeksema ${ }^{6}$ showed that ruminators committed more perseverative errors than non-ruminators on the Wisconsin card sorting test (WCST), ${ }^{7}$ and a similar phenomenon is seen in non-depressed people where so-called stimulus independent thoughts (SITs) ${ }^{8}$ interfere with the performance of executive tasks such as random number generation. ${ }^{9}$ Such studies, however, show only an association between executive dysfunction and concurrent off task cognitions. A more direct approach would be to manipulate rumination experimentally.

By either triggering rumination before the executive task, or inhibiting rumination with a distraction task before the executive task, we sought to test two hypotheses using random number generation as a brief but sensitive measure of executive function.

\section{HYPOTHESIS 1}

In depression, the central executive either functions less efficiently or has reduced capacity, leading to impairment on executive tasks even in the absence of rumination.

\section{HYPOTHESIS 2}

The central executive is essentially normal in depressed patients. Rumination, once triggered, draws resources from the executive interfering with concurrent capacity demanding cognitive tasks. In the absence of rumination, however, executive function is normal. Because depressed patients ruminate habitually, ${ }^{35}$ even in the absence of specific encouragement, and distraction seems to temporarily block such rumination, ${ }^{3}$ this hypothesis predicts that the distraction condition would improve random number generation performance in the depressed group.

\section{METHOD}

\section{Participants}

Fourteen depressed (male:female 6:8; mean age 42.9 (SD 10.0) years, mean duration of current depression 17.2 (SD 20.8) months; mean number of depressions 5.2 (SD 3.6); $78.6 \%$ prescribed antidepressant medication) and 14 nondepressed volunteers (male:female 4:10; mean age 36.2 (SD 13.1) years) were recruited by press advertisements. (Originally, 15 depressed participants were recruited but one did not seem to understand the random number generation task, producing count scores more than 3 SDs from the mean. With this participant excluded, the distribution of the sample was normally distributed. All data and analyses are reported with this outlier excluded.) All of the depressed and none of the non-depressed participants met criteria for current major depressive disorder (DSM-III-R), ${ }^{10}$ assessed using a standard instrument. ${ }^{11}$ The non-depressed participants had no history of depression within the past 5 years. The groups did not differ in age $\left(t(1,26)=-1.5\right.$, NS). $\chi^{2}$ Analyses showed that the groups did not differ in sex or in level of education.

\section{Materials and procedure}

Before and after induction (see below), participants rated their current mood on a 0-100 scale ranging from 0 "I do not feel at all despondent" to 100 "I feel extremely despondent". ${ }^{12}$

Abbreviations: WCST, Wisconsin card sorting test; SITs, stimulus independent thoughts; BDI, Beck depression inventory; RRS, ruminative responses scale; RTF, ruminative thought form 
Table 1 Means (SD) for random number generation count score, current mood, and rumination

\begin{tabular}{llllll}
\hline \multirow{2}{*}{ Variable } & \multicolumn{2}{l}{ Depressed } & & \multicolumn{2}{l}{ Non-depressed } \\
\cline { 2 - 3 } \cline { 5 - 6 } & Rumination Distraction & & Rumination & Distraction \\
\hline Count score & $68.8(44.7)$ & $48.4(23.3)$ & & $45.5(17.3)$ & $48.1(16.5)$ \\
Despondency & $64.6(23.7)$ & $55.8(15.3)$ & & $34.9(22.2)$ & $29.7(24.7)$ \\
Rumination & $33.5(16.9)$ & $28.9(15.4)$ & & $24.0(5.4)$ & $25.4(6.8)$ \\
\hline
\end{tabular}

Before the experiment, depression was assessed using the Beck depression inventory (BDI), ${ }^{13}$ and ruminative style using the ruminative responses scale (RRS) from the response style questionnaire. ${ }^{4}$ After each random number generation test, the level of within task rumination was assessed using a 20 item questionnaire (RTF) which measured how often common ruminative thoughts ${ }^{14}$ occurred (on a scale of $0=$ never, $\mathrm{l}=$ once, $2=$ twice, $3=$ more than twice) .

\section{Rumination and distraction inductions}

Before the random number generation tests (see below), participants performed either a rumination or distraction induction ${ }^{15}$ in counterbalanced order, within each group. In the rumination condition, participants focused on written items that were self and emotion focused-for example, "Think about what your feelings might mean". In the distraction condition, participants focused on items that were externally focused and unrelated to symptoms or feelings-for example, "Think about the shape of a large black umbrella". Before each induction, participants spent 5 minutes thinking about a recent difficulty to encourage a dysphoric mood. This phase was included because differential effects of rumination and distraction have only been found in the context of a dysphoric mood. ${ }^{315}$

\section{Random number generation task}

Participants were asked to say the numbers 1 to 9 in random order 100 times at the rate of one number a second, paced by a metronome. The concept of randomness was explained using standard procedures. ${ }^{16}$ Two trials were completed after each experimental condition. Recent research ${ }^{17}$ suggests that the count score ${ }^{18}$ is a highly sensitive measure of randomness. This measures the tendency to count in series (for example, $1-2-3,8-7-6-5)$, and is calculated as the sum of the squared length of each sequence of two or more.

\section{Design}

A 2 (group: depressed, non-depressed) $\times 2$ (condition: rumination, distraction) design was used. To ensure that any effect on random number generation was not due simply to change in despondent mood, the count scores were first adjusted using despondent mood as a covariate.

\section{RESULTS}

Table I displays the means (SD) for the count scores and mood measures. The groups differed significantly in BDI (depressed 25.1(6.8); non-depressed $3.9(3.4) ; F(1,27)=109.7$, p < 0.001) and RRS (depressed 63.0 (10.9); non-depressed 40.6 (10.6); F $(1,24)=26.9, \mathrm{p}<0.001)$.

\section{Effects of inductions on count scores}

An analysis of variance (ANOVA) disclosed a significant group $\times$ condition interaction $(F(1,26)=5.59, \mathrm{p}<0.03)$ reflecting higher count scores in the rumination induction, compared with the distraction induction in the depressed group $(t(13)=2.24, \mathrm{p}<0.05)$, but not in the non-depressed group $(t(13)=-0.76, \mathrm{p}=0.46$, table 1$)$.

\section{Effects of inductions on current mood}

An ANOVA showed that the depressed group were more despondent overall initially (depressed group 57.1 (SD 16); non-depressed $22.6 \quad(18.2) ; F \quad(1,26)=28.0, p<0.001)$. A significant condition $\times$ time interaction was also seen, reflecting an increase in despondency across groups after the rumination induction (before 39.0 (SD 23.0); after 49.7 (27.1)) but not the distraction induction (before 43.2 (25.9); after 42.7 (24.1); $F(1,26)=4.8, \mathrm{p}<0.05)$. There were no other significant main effects or interactions, with the inductions having similar effects on despondent mood in both groups.

\section{Effects of inductions on ruminative thoughts}

An ANOVA of the RTF scores showed a significant group $\times$ condition interaction $(F(1,26)=5.78, \mathrm{p}<0.03)$. This reflected a significant increase in ruminations in the depressed group after the rumination induction compared with distraction $(t(1,13)=2.1, \mathrm{p}<0.03$, one tailed $)$ but not in the non-depressed group $(t(1,13)=-1.21, \mathrm{p}=0.25$, table 1$)$.

\section{DISCUSSION}

The results suggest that rumination in depression occupies central executive resources also required for random number generation, and, specifically, for the suppression of the habitual counting tendency. Compared with distraction, rumination led to both higher count scores ( less random) and increased self report of ruminative thoughts in the depressed group, but not in the non-depressed group. This finding is consistent with the previously found association between ruminative style and perseverative errors on the WCST. ${ }^{6}$

Critically, in the distraction induction where ruminative thoughts were minimised, the depressed group was indistinguishable in their random number generation performance from the non-depressed group (table 1). This suggests that depressed patients do not have a fundamental deficit in central executive functioning, at least as it relates to the random number generation task. The results support the suggestion $^{19}$ that the association between rumination and executive function is due to rumination reducing executive capacity.

In terms of specific cognitive processes, the increased counting tendency in ruminating depressed patients suggests that ruminative thoughts occupy executive resources involved in the suppression of prepotent responses. Prepotent responses include the tendency to count in sequence, to respond in a manner congruent with a previous rule on the WCST, or to activate highly accessible thoughts or memories.

As expected, the results showed that the rumination induction produces ongoing ruminative thoughts in the depressed group for the duration of the random number generation task but not in the non-depressed group. Presumably, although non-depressed participants can voluntarily adopt a ruminative style, this is not their habitual thinking style (as indicated by their low RRS scores), and does not persist once they move onto another task. However, in depressed participants, rumination is a more habitual response, and the initial rumination induction may have triggered off pre-existing ruminative routines, whereas distraction temporarily blocked such routines.

We have assumed that the interference between rumination and random number generation and WCST is due to competition for processing capacity. However, interference can occur when the tasks share any processing stages, from encoding to output, even if those processes are not capacity limited.$^{20}$ Such "structural interference" might account for the impact of rumination on tasks dependent on immediate phonological encoding and retrieval processes, ${ }^{21}{ }^{22}$ such as the overt vocalisation during random number generation, or covert verbal processing during the WCST. However, previous research has shown that random number generation counting tendency is also influenced by a complex visuomotor tracking 
task with no verbal component ${ }^{23}$ supporting the capacity interference mechanism. Further research needs to assess the impact of rumination on a range of executive capacity demanding tasks, including those with minimal structural similarities.

However, whatever the source of the interference, the results have broader implications for our understanding of cognitive deficits in depression. Although we do not propose that rumination accounts for all findings of executive or other neuropsychological dysfunction in depression, the results suggest that rumination may play a significant part in some patients on some tasks. Future studies need to account for, or at least assess, levels of concurrent ruminative thinking. We have also shown that ruminative thinking is reversible, at least temporarily, by brief interventions such as distraction. The use of such interventions may allow for a better assessment of the true neurocognitive deficits associated with the prefrontal changes found in depression.

\section{Authors' affiliations}

E Watkins, R G Brown, Department of Psychology, Institute of Psychiatry, Kings College, London, UK

Correspondence to: Dr E Watkins, Department of Psychology, Institute of Psychiatry, De Crespingny Park, Denmark Hill, London SE5 8AF, UK

Received 3 April 2001

In revised form 5 October 2001

Accepted 31 October 2001

\section{REFERENCES}

1 Elliott R. The neuropsychological profile in unipolar depression. Trends in Cognitive Science 1998:2:447-84.

2 Beck AT. Cognitive therapy and the emotional disorders. New York: International Universities Press, 1976.

3 Nolen-Hoeksema S. Responses to depression and their effects on the duration of depressive episodes. J Abnorm Psychol 1991;100:569-82.

4 Nolen-Hoeksema S, Morrow J. A prospective study of depression and posttraumatic stress symptoms after a natural disaster: the 1989 Loma Prieta earthquake. J Pers Soc Psychol 1991;61:115-21.
5 Roberts JE, Gilboa E, Gotlib IH. Ruminative response style and vulnerability to episodes of dysphoria: Gender, neuroticism, and episode duration. Cognitive Therapy and Research 1998;22:401-23.

6 Davis RN, Nolen-Hoeksema S. Cognitive inflexibility among ruminators and nonruminators. Cognitive Therapy and Research 2000;24:699711 .

7 Heaton RK, Chelune GJ, Talley JL, et al. Wisconsin card sorting test manual: revised and expanded. Odessa, FL: Psychological Assessment Resources, 1993

8 Singer JL. Sampling on-going consciousness and emotional experiences: Implications for health. In: M Horowitz, ed. Psychodynamics and cognition. Chicago, IL: University of Chicago Press, 1988.

9 Teasdale JD, Dritschel BH, Taylor M, et al. Stimulus-independent thought depends on central executive resources. Memory and Cognition 1995;23:551-9.

10 American Psychiatric Association. Diagnostic and statistical manual of mental disorders, 3rd ed revised. Washington, DC: APA, 1987.

11 Spitzer RL, Williams JBW, Gibbon M, et al. SCID user's guide for the structured clinical interview for DSM-IIIR. Washington DC: American Psychiatric Press, 1990.

12 Teasdale JD, Taylor R, Fogarty SJ. Effects of induced elation depression on the accessibility of memories of happy and unhappy experiences. Behaviour Research and Therapy 1980;18:339-46.

13 Beck AT, Ward CH, Mendelson M, et al. An inventory for measuring depression. Arch Gen Psychiatry 1961;4:561-71.

14 Watkins E, Baracaia SB. Why do people in dysphoric moods ruminate? Personality and Individual Differences 2001:30:723-34.

15 Nolen-Hoeksema S, Morrow J. Effects of rumination and distraction on naturally occurring depressed mood. Cognition and Emotion 1993;7:561-70.

16 Horne RL, Evans FJ, Orne MT. Random number generation, psychopathology and therapeutic change. Arch Gen Psychiatry 1982;39:680-3.

17 Jahanshahi $M$, Profice $P$, Brown RG, et al. The effects of transcranial magnetic stimulation over the dorsolateral prefrontal cortex on suppression of habitual counting during random number generation. suppression of habitual countir

18 Ginsburg N, Karpiuk P. Random generation: analysis of the responses. Percept Mot Skills 1994;79:1059-67.

19 Williams JMG, Watts FN, Macleod C, et al. Cognitive psychology and emotional disorders. 2nd ed. Chichester, UK: Wiley, 1997.

20 Wickens CD. The structure of attentional resources. In: RS Nickerson, ed. Attention and performance VIII. Hillsdale, NJ: Erlbaum, 1980.

21 Kuhl J, Helle P. Motivational and volitional determinants of depression: the degenerated-intention hypothesis. J Abnorm Psycho 1986;95:247-51

22 Hertel PT. Relation between rumination and impaired memory in dysphoric moods. J Abnorm Psychol 1998;107:166-72.

23 Brown RG, Soliveri P, Jahanshahi M. Executive processes in Parkinson's disease: random number generation and response suppression. Neuropsychologia 1998;36:1355-62. 